\title{
Optimal Routing Based on Super Topology in Hypercube WDM Networks
}

\author{
Peng-Jun Wan* $\quad$ Liwu Liu ${ }^{\dagger} \quad$ Yuanyuan Yang ${ }^{\ddagger}$
}

\begin{abstract}
Traditionally the routing in passive optical networks is based on an embedded regular virtual topology. However one important fact that has been neglected in the past is that the wavelength assignment to transceivers actually creates additional (logical) links not present in the virtual topology. Such side effect can be utilized to significantly reduce the number of hops between a pair of stations. This observation leads to the concept of super topology. This paper considers the hypercube as the embedded virtual topology. The ideas contained here are easily applicable to networks employing other virtual topologies as well. We present the structure of the super topology, the optimal routing algorithm, the distance between any pair of stations and the diameter in the super topology.
\end{abstract}

Keywords: WDM, optical network, routing, virtual topology, super topology, distance, diameter.

\section{Introduction}

Optical passive star couplers $[6,8]$ provide a simple medium to connect nodes in a local or metropolitan area network [11]. Figure 1 shows a typical Wavelength Division Multiplexing (WDM) passive optical network in which each node is connected to the star coupler via a pair of unidirectional fibers. Each station has a set of transmitters and receivers. Each

\footnotetext{
${ }^{*}$ Department of Computer Science, Illinois Institute of Technology, Chicago, IL 60616. Email: wan@cs.iit.edu.

†Department of Computer Science, Illinois Institute of Technology, Chicago, IL 60616. Email: liwuliu@cs.iit.edu.

$\ddagger$ Department of ECE, SUNY at Stony Brook, Stony Brook, NY 11794. Email: yang@ece.sunysb.edu.
}

transmitter (receiver) is tuned to a specific wavelength channel from which it transmits (receives) light signals into (from) an optical fiber. The light signals entering the star coupler are evenly divided among all the output ports. A transmission from one station to another station is accomplished by tuning a transmitter of the sending station and a receiver of the receiving station to the same wavelength. Transmissions with different wavelength channels can take place simultaneously. If the number of wavelength channels is less than the number of transmitters (or receivers), the wavelength channels can be shared among them in the time-division multiplexing manner, which results in Time and Wavelength Division Multiplexing (TWDM) media access protocols [5, 7].

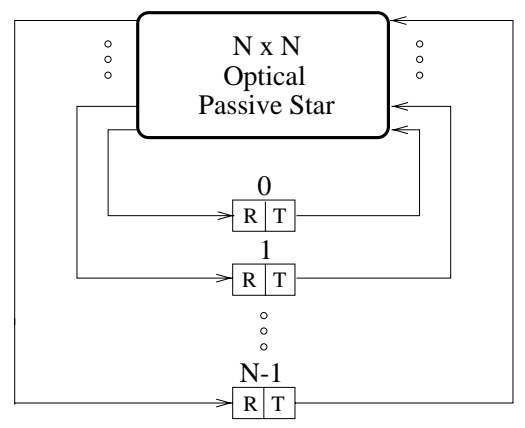

Figure 1: An $N$-node optical passive star network

The transceivers at each station could be either fixed or tunable. The fixed transceivers have several advantages over the tunable transceivers. Currently, the tunable transceivers cost much more than the fixed transceivers. The tuning speed of the tunable transceivers is very slow comparing to the transmis- 
sion speed of optical fibers and is inverse to its tunable range. Furthermore, the tunable transceivers require accurate pre-transmission coordination. However, the fixed transceivers also have some disadvantages. The main disadvantages include that they are bulky and the transmission concurrence may be limited by the fixed transceivers. For these reasons, passive optical networks with a small number of fixed wavelength transceivers have been proposed in the past. As show in [13], this configuration can greatly improve the performance while being able to emulate the tunability of the tunable transceivers without suffering tuning delay.

Traditionally, the routing in a passive optical network with a small number of fixed-wavelength transceivers adopts the same routing algorithm developed in the regular interconnection topology that is embedded in the passive optical network $[9,10]$. However, one important fact that has been ignored in the past is that the process to realize or embed the regular interconnection topology actually creates some by-products, the additional (logical) links not present in the original regular interconnection topology. Thus the actual logical interconnection pattern is a super graph of the embedded regular interconnection topology, and hence is referred to as super topology. Because of the better connectivity in the super topology, such side-effect can be exploited to reduce the distance in terms of the number of hops among stations. This can be illustrated in the following simplest example. Consider a passive optical network of eight stations into which a 3 -cube is embedded as follows. Each station has a single transmitter and a single receiver. The transmitters at stations $000,011,101,110$ and the receivers at stations 001,010,100,111 are assigned with wavelength $\lambda_{0}$, while the receivers at stations $000,011,101,110$ and the transmitters at stations 001, 010, 100, 111 are assigned with wavelength $\lambda_{1}$. Such wavelength assignment realizes the 3 -cube. Now we consider the routing from station 000 to station 111 . If the routing is simply based on the routing in the 3 -cube, then the shortest distance is three hops. However, notice that the transmitter at station 000 and the receiver at the station 111 have the same wavelength $\lambda_{0}$, the station 000 can talk to the station 111 directly, and therefore their distance is just one. A graph theoretic explanation to this improvement is the difference between the embedded 3-cube and the actual super topology. Figure 2 shows the super topology of the above wavelength assignment. It is the super graph of 3-cube. In addition to the links in the 3 -cube, four additional links are present in the super topology: the link between 000 and 111, the link between 001 and 110, the link between 010 and 101, and the link between 100 and 011. It's easy to see that the diameter of this super topology is two, in contrast to the fact that the diameter of the 3 -cube is three.

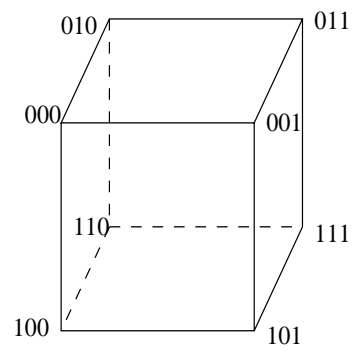

(a)

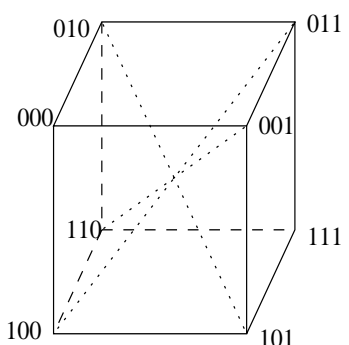

(b)
Figure 2: The super topology of the embedded 3cube.

The above observation leads to the question on how better is the super topology than the original regular interconnection topology in terms of the network properties such as routing, the load balancing and fault tolerance. This paper characterizes the super topologies in passive optical hypercube networks with various numbers of transceivers. The algorithms for optimal routing are presented. The difference between any pair of stations and the diameters are also given in terms of the number of hops.

The remaining of this paper is arranged as follows. Section 2 introduces some key concepts such as transmission graph, subnetworks and their relations to the super topology. Section 3 presents the structure of the super topology, the optimal routing algorithm, the distance between and pair of stations and the diameter in the super topology when the number of transmitters at each station is equal to the number of receivers at each station. Section 4 studies the 
same issues when the number of transmitters at each station is less than the number of receivers at each station. Finally Section 5 concludes the paper.

\section{Preliminaries}

To embed a given interconnection topology into a passive optical network, one must first partition the outgoing links at each station into a number of groups with one for each transmitter; and similarly, partition the incoming links at each station into a number of groups with one for each receiver. The partition determines a transmission graph, a bipartite digraph whose vertex sets are all transmitters and all receivers, and there is a link from a transmitter to a receiver if and only if they are responsible for one common link in the interconnection topology to be realized. It's obvious that the number of links in the transmission graph is the same as that in the interconnection topology.

The partition imposes a constraint on the wavelength assignment of the transmitters and receivers as explained below. Since the transmitters and receivers are fix-tuned, any transmitter (receiver) and its adjacent receivers (transmitters) in the transmission graph are forced to have the same wavelength channels of the transmitter (receiver). Therefore any pair of transceivers must have the same wavelength channel if there is path between them assuming the links in the transmission graph are bidirectional. This key observation leads to the concept of subnetworks. In the transmission graph, a set of transmitters and receivers form a subnetwork if there is a path between any two of them if we ignore the unidirectional nature of the links ${ }^{1}$. Thus all transmitters and receivers in the same subnetwork must have the same wavelength channel. In this paper, we assume that the number of transceivers are selected such that each subnetwork has a unique wavelength.

The structure of subnetworks provides a way to determine the super topology of a wavelength assignment. In general, there is a link from station $a$ to

\footnotetext{
${ }^{1}$ The subnetwork concept is similar to the concept of connected component in the graph theory. The only difference is that here we ignore the unidirectional nature of the links.
}

station $b$ in the super topology if and only if $a$ has a transmitter and $b$ has a receiver which are in the same subnetwork. The characterization of the structure of subnetworks is reported in a separate paper [14]. In this paper, we first determine from the structure of subnetworks the set of neighbors of each station and the nodal degree in the super topology. After that we present the optimal routing algorithm in the super topology. Finally we give the analytic expression for the diameter in terms of the number of hops in the super topology.

Due to the symmetry of the hypercube, swapping the number of transmitters and receivers does not change the connectivity of the super topology. Thus we only consider the cases that the number of transmitters is no more than the number of receivers. Section 3 studies the configuration in which the number of transmitters is equal to the number of receivers. Section 4 studies the configuration in which the number of transmitters is less than the number of receivers.

For the simplicity of discussion, we first introduce some notations. We use $T$ and $R$ to denote the number of transmitters and the number of receivers respectively at each station. For any $S \subseteq$ $\{0,1, \cdots, n-1\}$ and any $n$-bit binary number $a$, we use $\left.a\right|_{S}$ to denote the $|S|$-bit binary number consisting of the bits of $a$ at positions in $S$, and $\left.a\right|_{S}$ to denote the $(n-|S|)$-bit binary number consisting of the bits of $a$ at positions not in $S$. For any two stations $a$ and $b$, we use $H(a, b)$ to denote their distance in terms the number of hops in the super topology.

\section{Case 1: $T=R$}

\subsection{Super Topology}

We begin with the simplest case that $T=R$. The $n$ dimensions are consecutively partition into $T$ groups $D_{0}, D_{1}, \cdots, D_{T-1}$ with

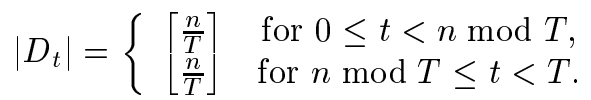

The transmitter $(a, t)$ is responsible for the outgoing links at $a$ along dimensions in $D_{t}$, and the receiver 
$(a, r)$ is responsible for the incoming links at $a$ along dimensions in $D_{r}$. Then according to [14], the subnetwork that the transmitter $(a, t)$ belongs to consist of all transmitters

$$
\left\{(b, t):\left.(a \oplus b)\right|_{D_{t}} \text { is even and }\left.(a \oplus b)\right|_{\overline{D_{t}}}=0\right\}
$$

and all receivers

$$
\left\{(b, t):\left.(a \oplus b)\right|_{D_{t}} \text { is odd and }\left.(a \oplus b)\right|_{\overline{D_{t}}}=0\right\} .
$$

Thus in the super topology, the adjacent stations of the station $a$ is

$$
\bigcup_{t=0}^{T-1}\left\{b:\left.(a \oplus b)\right|_{D_{t}} \text { is odd and }\left.(a \oplus b)\right|_{\overline{D_{t}}}=0\right\} \text {. }
$$

So the nodal degree of each station in the super topology is

$$
\begin{aligned}
& \sum_{t=0}^{T-1} 2^{\left|D_{t}\right|-1} \\
& =(n \bmod T) 2^{\left\lceil\frac{n}{T}\right\rceil-1}+(T-n \bmod T) 2^{\left\lfloor\frac{n}{T}\right\rfloor-1} \\
& =(T+n \bmod T) 2^{\left\lfloor\frac{n}{T}\right\rfloor-1} .
\end{aligned}
$$

As

$$
(T+n \bmod T) 2^{\left\lfloor\frac{n}{T}\right\rfloor-1}=n
$$

if and only if $T>\frac{n}{2}$, each station has more neighbors in the super topology if $T \leq \frac{n}{2}$, and thus may reduce the distances between some nodes.

\section{$3.2 \quad$ Optimal Routing}

Now let's look at the optimal routing in the super topology. The routing from station $a$ to station $b$ is equivalent to changing the bits of $a$ to the bits of $b$ according to certain rules. In the super topology with $T=R$, at each step any odd number of bits at positions in some $D_{t}$ are allowed to be reversed simultaneously. Recall that in the original $n$-cube, only one bit can be changed at a time. Thus the distance in terms of the number of steps/hops to change $a$ to $b$ should be smaller.

Note that at each step the reversing of bits at positions in some $D_{t}$ has no impact on the bits in other positions. Thus to change the bits of $a$ at positions in $D_{t}$ to the bits of $b$ at positions in $D_{t}$, we only have to look at $\left.(a \oplus b)\right|_{D_{t}}$. Suppose that $\left.(a \oplus b)\right|_{\overline{D_{t}}}=0$ and $\left.(a \oplus b)\right|_{D_{t}} \neq 0$. Then

- if $\left.(a \oplus b)\right|_{D_{t}}$ is odd, then a single hop is needed from station $a$ to station $b$;

- if $\left.(a \oplus b)\right|_{D_{t}}$ is even, then two hops are needed from station $a$ to station $b$;

Therefore, the routing can be performed sequentially for each $0 \leq t<T$. The optimal routing given in Table 1 is very similar to the well-known $Z$-routing in the hypercube. It is given in the recursive format for the simplicity of description.

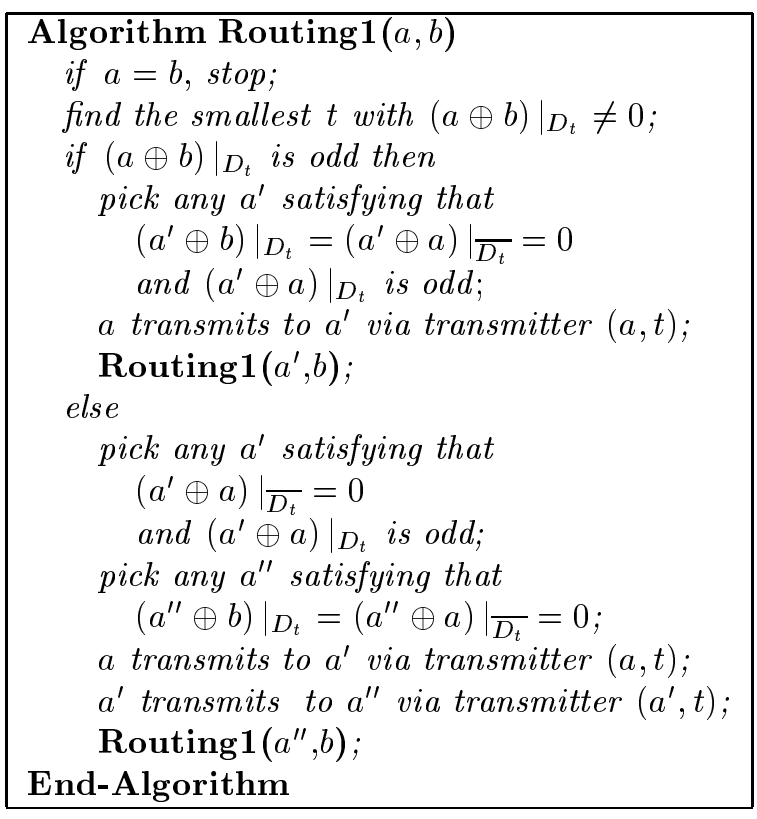

Table 1: Optimal routing from $a$ to $b$ when $T=R$.

\subsection{Distance And Diameter}

In the next we will study the distance between any pair of stations and the diameter of the super topology. 
For any binary number $a$ and amy $0 \leq t<T$, we define $h_{t}(a)$ as follows.

- if $\left.a\right|_{D_{t}}=0$, then $h_{t}(a)=0$;

- if $\left.a\right|_{D_{t}} \neq 0$ and $\left.a\right|_{D_{t}}$ is odd, then $h_{t}(a)=1$;

- if $\left.a\right|_{D_{t}} \neq 0$ and $\left.a\right|_{D_{t}}$ is even, then $h_{t}(a)=2$.

Then the distance between the station $a$ and the station $b$ is given by

$$
H(a, b)=\sum_{t=1}^{T-1} h_{t}(a \oplus b) .
$$

It's easy to see that for any $0 \leq t<T$, the maxima of $h_{t}(a)$ is two if $\left|D_{t}\right|>1$, and is one if $\left|D_{t}\right|=1$. Therefore, if $n \geq 2 T$, the diameter is equal to $2 T$. Now we assume that $n<2 T$. For any $0 \leq t<n-T$, $\left|D_{t}\right|=2$ and thus the maxima of $h_{t}(a)$ is two. For any $n-T \leq t<T,\left|D_{t}\right|=1$ and thus the maxima of $h_{t}(a)$ is one. Therefore the diameter is

$$
2(n-T)+(2 T-n)=n .
$$

In summary, when $T=R$, the diameter of the super topology is $\min \{n, 2 T\}$. Hence the fewer the number of transmitters/receivers, the shorter the diameter. However, the fewer number of transmitters may cause larger number of transmitters/receivers in each subnetwork and result in longer channel access delay. The worst number of time slots can be as large as

$$
2 T \cdot 2^{\left\lceil\frac{n}{T}\right\rceil-1}=T 2^{\left\lceil\frac{n}{T}\right\rceil}
$$

which might decrease as $T$ increase.

\section{Case 2: $T<R$}

\subsection{Super Topology}

Now we consider the configuration with $0<T<R<$ $n$. The $n$ dimensions are consecutively partitioned into $T$ groups $D_{0}, D_{1}, \cdots, D_{T-1}$ with

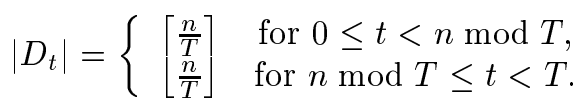

The transmitter $(a, t)$ is responsible for the outgoing links at $a$ along dimensions in $D_{t}$. For each $0 \leq t<$ $R \bmod T, D_{t}$ is further consecutively partitioned into $\left\lceil\frac{R}{T}\right\rceil$ subgroups

$$
D_{t, 0}, D_{t, 1}, \cdots, D_{t,\left\lceil\frac{R}{T}\right\rceil-1}
$$

of arbitrary sizes, and the receiver $\left(a, t\left\lceil\frac{R}{T}\right\rceil+i\right)$ is responsible for the incoming links at $a$ along dimensions in $D_{t, i}$ for any $0 \leq i<\left\lceil\frac{R}{T}\right\rceil$. For each $R \bmod T \leq t<T, D_{t}$ is further consecutively partitioned into $\left\lfloor\frac{R}{T}\right\rfloor$ subgroups

$$
D_{t, 0}, D_{t, 1}, \cdots, D_{t,\left\lfloor\frac{R}{T}\right\rfloor-1}
$$

of arbitrary sizes, and the receiver $(a, R \bmod T+$ $\left.t\left\lfloor\frac{R}{T}\right\rfloor+i\right)$ is responsible for the incoming links at $a$ along dimensions in $D_{t, i}$ for any $0 \leq i<\left\lfloor\frac{R}{T}\right\rfloor$. Then if $0 \leq t<R \bmod T$ the subnetwork that the transmitter $(a, t)$ belongs to consists of all transmitters

$$
\begin{gathered}
\left\{(b, t):\left.(a \oplus b)\right|_{\overline{D_{t}}}=0,\left.(a \oplus b)\right|_{D_{t, i}}\right. \text { is even } \\
\text { for any } \left.0 \leq i<\left\lceil\frac{R}{T}\right\rceil\right\} .
\end{gathered}
$$

and all receivers

$$
\begin{aligned}
& \bigcup_{i=0}^{\left\lceil\frac{R}{T}\right\rceil-1}\left\{\left(b, t\left\lceil\frac{R}{T}\right\rceil+i\right):\left.(a \oplus b)\right|_{\overline{D_{t}}}=0,\left.(a \oplus b)\right|_{D_{t, i}}\right. \\
& \left.\quad \text { is odd and }\left.(a \oplus b)\right|_{D_{t, j}} \text { is even for any } j \neq i\right\} .
\end{aligned}
$$

If $R \bmod T \leq t<T$ the subnetwork that the transmitter $(a, t)$ belongs to consists of all transmitters

$$
\begin{gathered}
\left\{(b, t):\left.(a \oplus b)\right|_{\overline{D_{t}}}=0,\left.(a \oplus b)\right|_{D_{t, i}}\right. \\
\text { is even for any } \left.0 \leq i<\left\lfloor\frac{R}{T}\right\rfloor\right\}
\end{gathered}
$$

and all receivers

$$
\begin{aligned}
\bigcup_{i=0}^{\left\lfloor\frac{R}{T}\right\rfloor-1} & \left\{\left(b, R \bmod T+t \mid \frac{R}{T}\right\rfloor+i\right):\left.(a \oplus b)\right|_{\overline{D_{t}}}=0, \\
& \left.(a \oplus b)\right|_{D_{t, i}} \text { is odd, and }\left.(a \oplus b)\right|_{D_{t, j}} \\
& \text { is even for any } j \neq i\} .
\end{aligned}
$$


Thus in the super topology, the adjacent stations of the station $a$ is

$$
\begin{gathered}
\bigcup_{t=0}^{R \bmod T-1} \bigcup_{i=0}^{\left\lceil\frac{R}{T}\right\rceil-1}\left\{b:\left.(a \oplus b)\right|_{\overline{D_{t}}}=0,\left.(a \oplus b)\right|_{D_{t, i}}\right. \text { is } \\
\bigcup_{t=R \bmod T}^{T-1} \bigcup_{i=0}^{\left\lceil\frac{R}{T}\right\rceil-1}\left\{b:\left.(a \oplus b)\right|_{D_{t, j}} \text { is even } \forall j \neq i\right\} \quad \cup \\
\text { odd, } \left.\left.(a \oplus b)\right|_{D_{t, j}} \text { is even } \forall j \neq i\right\}
\end{gathered}
$$

So the nodal degree of each station in the super topology is

$$
\sum_{t=0}^{R \bmod T-1}\left\lceil\frac{R}{T}\right\rceil 2^{\left|D_{t}\right|-\left\lceil\frac{R}{T}\right\rceil}+\sum_{t=R \bmod T}^{T-1}\left\lfloor\frac{R}{T}\right\rfloor 2^{\left|D_{t}\right|-\left\lfloor\frac{R}{T}\right\rfloor}
$$

which can be simplified as follows:

- If $n \bmod T=R \bmod T$, then the degree is

$$
R 2^{\frac{n-R}{T}} \text {. }
$$

- If $n \bmod T<R \bmod T$, then the degree is

$$
R 2^{\left\lceil\frac{n-R}{T}\right\rceil}+(n \bmod T-R \bmod T)\left\lceil\frac{R}{T}\right\rceil 2^{\left\lfloor\frac{n-R}{T}\right\rfloor} .
$$

- If $n \bmod T>R \bmod T$, then the degree is

$$
\left(R+((n-R) \bmod T)\left\lfloor\frac{R}{T}\right\rfloor\right) 2^{\left\lfloor\frac{n-R}{T}\right\rfloor} .
$$

\subsection{Optimal Routing}

Now let's look at the optimal routing in the super topology. We again treat the routing from $a$ to $b$ as a number of steps to change the bits of $a$ to the bits of $b$. For any $0 \leq t<T$, any odd number of bits at positions in some $D_{t, i}$ and any even number of bits at positions in any $D_{t, j}$ with $j \neq i$ are allowed to be reversed simultaneously within a single step. Note that at each step the reversing of bits at positions in some $D_{t}$ has no impact on the bits in other positions. Thus to change the bits of $a$ at positions in $D_{t}$ to the bits of $b$ at positions in $D_{t}$, we only have to look at $\left.(a \oplus b)\right|_{D_{t}}$. Suppose that $\left.(a \oplus b)\right|_{\overline{D_{t}}}=0$ and $\left.(a \oplus b)\right|_{D_{t}} \neq 0$. Then
- if $\left.(a \oplus b)\right|_{D_{t, i}}$ is even for all $i$, then two hops are needed from station $a$ to station $b$;

- otherwise, the minimal number of hops required from station $a$ to station $b$ is equal to the number of $i$ 's with odd $\left.a\right|_{D_{t, i}}$.

Such procedure can be repeated sequentially for each $0 \leq t<T$. The recursive version of an optimal routing algorithm is given in Table 2 .

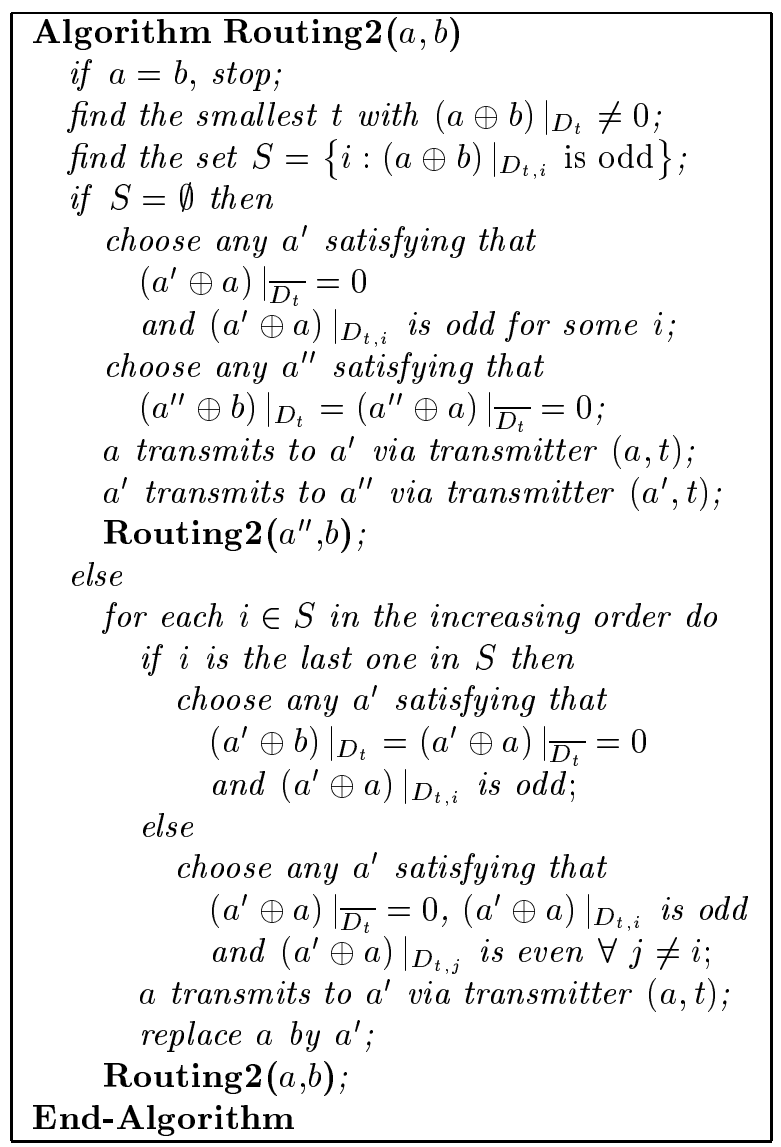

Table 2: Optimal routing from $a$ to $b$ when $T<R$.

\subsection{Distance And Diameter}

For any binary number $a$ and any $0 \leq t<T$, we define $h_{t}(a)$ as follows. 
- if $\left.a\right|_{D_{t}}=0$, then $h_{t}(a)=0$;

- if $\left.a\right|_{D_{t}} \neq 0$ and $\left.a\right|_{D_{t, i}}$ is even for all $i$, then $h_{t}(a)=2$;

- if $\left.a\right|_{D_{t}} \neq 0$ and $\left.a\right|_{D_{t, i}}$ is odd for some $i$, then $h_{t}(a)$ is equal the number of $i$ 's satisfying that $\left.a\right|_{D_{t, i}}$ is odd.

Then the distance between the station $a$ and the station $b$ is given by

$$
H(a, b)=\sum_{t=1}^{T-1} h_{t}(a \oplus b) .
$$

Thus the diameter is the sum of the maxima of $h_{t}(a)$ over all $0 \leq t<T$. In general, if the number of receivers at each station that are responsible for the links along dimensions in $D_{t}$ is more than one, the maxima of $h_{t}(a)$ is equal to such number. If there is only one receiver at each station that is responsible for the links along dimensions in $D_{t}$, then the maxima of $h_{t}(a)$ is equal to two if $\left|D_{t}\right|>1$ and equal to one if $\left|D_{t}\right|>1$.

If $R \geq 2 T$, then $\left[\frac{R}{T}\right\rceil \geq\left\lfloor\frac{R}{T}\right\rfloor \geq 2$. Thus for any $0 \leq t<T$, there are at least two receivers that are responsible for the links along dimensions in $D_{t}$. This implies that the maxima of $h_{t}(a)$ is equal to the number of receivers at each station that are responsible for the links along dimensions in $D_{t}$. So the diameter is equal to $R$, the total number of receivers at each station.

If $T<R<2 T \leq n$, then $\left\lceil\frac{n}{T}\right\rceil \geq\left\lfloor\frac{n}{T}\right\rfloor \geq 2$. For $0 \leq t<R-T$, there are exactly two receivers that are responsible for the links along dimensions in $D_{t}$, hence the maxima of $h_{t}(a)$ is 2 . For $R-T \leq t<T$, $\left|D_{t}\right| \geq 2$ while there is only one receiver at each station that is responsible for the links along dimensions in $D_{t}$. So the maxima of $h_{t}(a)$ is also equal to two. Therefore the diameter is $2 T$.

If $T<R<n<2 T$, we show that the diameter is $n$. In fact, for $0 \leq t<R-T,\left|D_{t}\right|=\left\lceil\frac{n}{T}\right\rceil=2$ and there are exactly two receivers that are responsible for the two links along dimensions in $D_{t}$. Hence the maxima of $h_{t}(a)$ is 2. For $R-T \leq t<n-T$, $\left|D_{t}\right|=\left\lceil\frac{n}{T}\right\rceil=2$ but there is only one receiver at each station that is responsible for the two links along dimensions in $D_{t}$. So the maxima of $h_{t}(a)$ is also equal to two. For $N-T \leq t<T-1,\left|D_{t}\right|=\left\lfloor\frac{n}{T}\right\rfloor=1$ and there is only one receiver at each station that is responsible for the link along dimension in $D_{t}$. So the maxima of $h_{t}(a)$ is equal to one. Therefore the diameter is

$$
2(R-T)+2(n-T)+(2 T-n)=n .
$$

In summary, when $0<T<R<n$, the diameter is equal to $\min \{n, \max \{R, 2 T\}\}$.

\section{Conclusion}

Traditionally the routing in passive optical networks is based on an embedded regular virtual topology. However the wavelength assignment that realizes the embedding actually creates a logical connectivity which can be represented as a super graph of the embedded virtual topology. This paper studies how to explore such side effect to reduce the number of hops between a pair of stations in a passive optical hypercube network. We present the structure of the super topology, the optimal routing algorithm, the distance between any pair of stations and the diameter in the super topology. The ideas and approaches contained in this paper are easily applicable to passive optical networks embedded into with other virtual topologies such as the de Bruijn graph [12], the star graph [1], and the rotator graph [4] as well.

\section{References}

[1] S.B. Akers, D. Harel, B. Krishnamurthy, "The Star Graph: An Attractive Alternative to The $n$ cube", Proc. Int. Conf. Parallel Processing, pp. 393-400, 1987.

[2] Brackett C.A., "On the Capacity of Multiwavelength Optical-Star Packet Switches", IEEE Lightwave Magazine, May 1991, pp. 33-37.

[3] Chen M.S., Dono N.R., Ramaswami R., "A Media Access-Protocol for Packet-Switched Wavelength Division Multiaccess Metropolitan Networks", IEEE Journal on Selected Areas in 
Communications, vol. 8, no. 6, Aug 1990, pp. 1048-1057.

[4] P.F. Corbett, "Rotator Graphs: AN Efficient Topology for Point-to-Point Multiprocessor Networks", IEEE Transactions on Parallel And Distributed Systems, Vol. 3, No. 5, Sep 1992. pp-622626.

[5] Dam T.Q., Williams K.A., Du D.H.C., "A Media-Access Protocol for Time and Wavelength Division Multiplexed Passive Star Networks", Technical Report 91-63, Computer Science Dept., University of Minnesota.

[6] Dragone C., "Efficient N x N Star Coupler Based on Fourier Optics", Electronics Letters, vol. 24, no. 15 , Jul 1988, pp. 942-944.

[7] Hluchyj M.G., Karol M.J., "ShuffleNet: An Application of Generalized Perfect Shuffles to Multihop Lightwave Networks", Journal of Lightwave Technology, vol. 9, no. 10, Oct 91, pp. 13861396.

[8] Linke R.A., "Frequency Division Multiplexed Optical Networks Using Heterodyne Detection", IEEE Network Magazine, vol. 3, no. 2, Mar 1989, pp. 13-20.

[9] B. Mukherjee, "WDM-based Local Lightwave Networks-Part I: Single-hop Systems", IEEE Network, vol. 6, pp. 12-27, May 1992.

[10] B. Mukherjee, "WDM-based Local Lightwave Networks-Part II: Multihop Systems", IEEE Network, vol. 6, pp. 20-32, July 1992.

[11] B. Mukherjee, Optical Communication Networks, New York: McGraw-Hill, July 1997.

[12] Sivarajan K., Ramaswami R., "Multihop Lightwave Networks Based on De Bruijn Graphs", INFOCOM 90, vol. 2, 1990, pp. 1001-1011.

[13] Wan P.-J., "Multichannel Optical Networks", Kluwer Acdemic Publishers, 1999 (to appear).

[14] Wan P.-J.,"Multi-Transceiver Passive Optical Networks", submitted for publication. 